\title{
Bubble CPAP: Can We Predict Success or Failure?
}

\author{
RAKESH SAHNI \\ Division of Neonatal-Perinatal Medicine, Department of Pediatrics, College of Physicians and Surgeons, Columbia University, 630 \\ W. 168th Street, New York, NY 10032, New York, NY, USA.rs62@columbia.edu
}

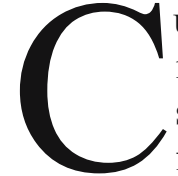

urrent modalities of ventilatory assistance in the management of respiratory distress syndrome (RDS) in preterm infants range from continuous positive airway pressure (CPAP) to various modes of mechanical ventilation. The use of CPAP has been associated with a lower incidence of chronic lung disease when used as the initial respiratory support. In contrast, mechanical ventilation has the potential to injure the airways and lung parenchyma.

Recently, there has been a renewed interest in gentle ventilation strategies, such as CPAP as a way to improve outcomes for very preterm infants. A wide variety of devices are used to deliver CPAP, including variable flow driver devices, single or binasal prongs where pressure is generated by a column of water (bubble CPAP) or a ventilator. Bubble CPAP (BCPAP) is appealing because of its simplicity and low cost. With this technique gas flows past the nasal device and the pressure is generated in the circuit by placing the distal limb of the CPAP circuit under a known depth of water that creates bubbles and pressure oscillations in the circuit. Gas flow is increased until continuous bubbling is achieved. It has been suggested that use of BCPAP in the poorly compliant lung may promote lung volume recruitment and augment the efficiency of gas mixing. The usefulness of BCPAP in the initial management of infants with RDS remains controversial as little data are available. Additionally, not all extremely premature infants with RDS are candidates for initial treatment with CPAP, and not all those who are given CPAP can be successfully managed with this modality.
The prospective observational study of Murki, et al.(1) reported in this issue of Indian Pediatrics evaluates the immediate outcome of preterm infants with RDS on BCPAP and identifies risk factors associated with its failure. The results emphasize the utility of a simple, inexpensive, and yet a powerful and effective technique of respiratory support, particularly suitable for neonatal units with limited resources. Although their study population included relatively larger and more mature infants, the clinical experience with BCPAP was very similar to that reported by us and others $(2,3)$.

In our clinical experience with the use of early nasal BCPAP as the initial mode of respiratory support for all spontaneously breathing infants with birth weight $\leq 1250 \mathrm{~g}$, we recently reported (2) that nasal BCPAP was successful in about half of the infants less than $800 \mathrm{~g}$ birth weight and over $80 \%$ in infants more than $800 \mathrm{~g}$. The overall CPAP success rate was $76 \%$. The use of early nasal BCPAP increased with increasing postmenstrual age (PMA). Over 26 weeks PMA, nasal BCPAP was initiated in over $90 \%$ of the infants, and was successfully maintained in most of them. Immaturity (PMA $<26$ weeks), small birth size (birth weight $<750 \mathrm{~g}$ ), need for positive pressure ventilation at delivery, and severity of RDS (as indicated by $\mathrm{AaDO}_{2}$ gradient $>180 \mathrm{mmHg}$ and severe RDS on initial chest $X$-ray) were strongly associated with early BCPAP failure. However, none of these factors had a positive predictive value above $55 \%$. In infants with birth weights $<1251 \mathrm{~g}$, the incidence of bronchopulmonary dysplasia (BPD) at 28 days and 36 weeks PMA was $21.1 \%$ and $7.4 \%$, respectively. Using the 
recently defined NIH criteria, the incidence of mild, moderate and severe BPD were $13.5 \%, 4.8 \%$ and $2.6 \%$, respectively(4). Similarly, in a recent large multicenter, randomized controlled trial, Morley, et al.(5), also reported that preterm infants $(n=610$, gestation: 25-28 wk and mean birth weight 960g) treated with early nasal CPAP versus those who were ventilated demonstrated similar, or slightly better outcomes (death or BPD).

There is a learning curve to the implementation of a nasal BCPAP based respiratory approach that requires team effort for success. The authors indicate that BCPAP for treatment of RDS was available to them for only six months before the onset of the study. Aly, et al.(6) have reported the impact of experience over time, with the use of early nasal BCPAP on outcome of extremely low birth weight infants. Similar to the Columbia experience, they demonstrated that in preterm infants $<1500 \mathrm{~g}$, nasal BCPAP could be initiated at very low gestational age. The early use of BCPAP management increased in the surviving infants over time, whereas the use of surfactant decreased and the incidence of BPD decreased from $33 \%$ to $6 \%$.

Although the recent debate on the use of either nasal CPAP or mechanical ventilation as the initial treatment of preterm infants with RDS is flawed by a lack of published data from large randomized clinical trials; emerging evidence suggests that BCPAP has a significant role in the clinical management of infants with RDS. However, further research is required to establish good early predictors of BCPAP success or failure in the management of RDS in preterm infants.

Funding: None.

Competing interests: None stated.

\section{REFERENCES}

1. Koti J, Murki S, Gaddam P, Reddy A, Reddy MDR. Bubble CPAP for respiratory distress syndrome in preterm infants. Indian Pediatr 2010; 47: 139-143.

2. Ammari A, Suri M, Milisavljevic V, Sahni R, Bateman D, Sanocka U, et al. Variables associated with early failure of nasal CPAP in very low birth weight infants. J Pediatr 2005; 147: 341-347.

3. De Klerk AM, De Klerk RK. Nasal continuous positive airway pressure and outcomes of preterm infants. J Paediatr Child Health 2001; 37: 161-167.

4. Sahni R, Ammari A, Suri MS, Milisavljevic V, Ohira-Kist K, Wung JT, et al. Is the new definition of bronchopulmonary dysplasia more useful? J Perinatol 2005; 25: 41-46.

5. Morley CJ, Davis PG, Doyle LW, Brion LP, Hascoet JM, Carlin JB; COIN Trial Investigators. Nasal CPAP or intubation at birth for very preterm infants. N Engl J Med 2008; 358: 700-708.

6. Aly H, Milner JD, Patel K, El-Mohandes AA. Does the experience with the use of nasal continuous positive airway pressure improve over time in extremely low birth weight infants? Pediatrics 2004; 114: 697-702. 